\title{
Enantioselective Separation of Chiral N1-Substituted-1H-pyrazoles: Greenness Profile Assessment and Chiral Recognition Analysis
}

\author{
Mohammed Farrag El-Behairy, Rasha M. Hassan, and Inas A. Abdallah* \\ Cite This: ACS Omega 2021, 6, 25835-25841 \\ Read Online
}

ABSTRACT: Two commercialized polysaccharide-based chiral stationary phases, Lux cellulose- 2 and Lux amylose-2, were examined for their chiral recognition ability on a set of 18 biologically active racemic 4,5-dihydro- $1 \mathrm{H}$-pyrazole derivatives by applying normal and polar organic elution modes. The results showed that all compounds were baseline-resolved with at least one of the used elution modes. The cellulose-based column was superior using polar organic mobile-phase compositions with analysis times close to $5 \mathrm{~min}$ and resolutions up to 18 , while the enantiomer-resolving ability of amylose-based columns was greater using the normal elution mode with analysis times close to $30 \mathrm{~min}$ and resolutions up to 30 . The competition between the analytes and the mobile phase constituents on H-bond interactions with the

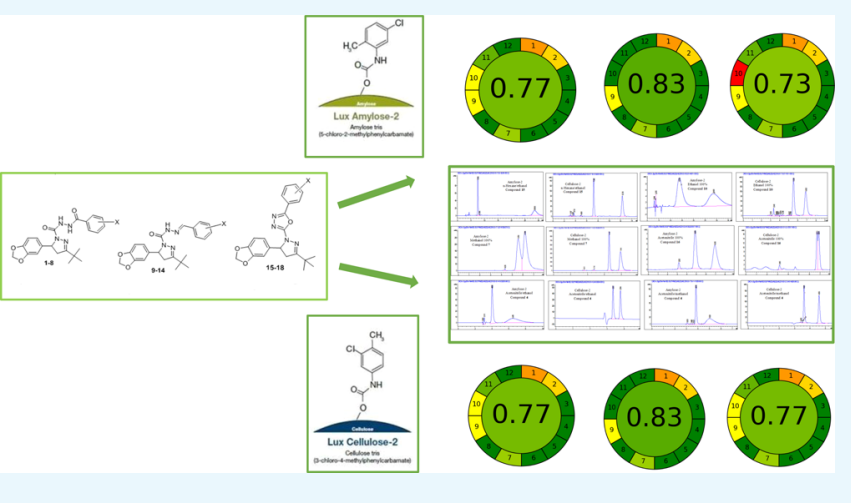
stationary phase has been discussed, and the impact of this competition on chiral recognition has been investigated. It was found that the polar organic mode is very beneficial for short run times and sharp peaks. The developed enantioselective high-performance liquid chromatography (HPLC) methods will be applied to monitor the stereoselective synthesis of compounds 1-18 or to develop preparative HPLC techniques for compounds 1-18, followed by stereospecific pharmacological studies for each enantiomer separately. Greenness profile assessment of the different elution solvents was carried out using the AGREE metric approach.

\section{INTRODUCTION}

Stereochemistry has become a key influencer in drug discovery and the pharmaceutical industry. Chirality is represented in up to $50 \%$ of the drug market, with nine of the best-selling drugs in 2018 chiral with two of them being commercialized as pure enantiomers. $^{1-3}$ Domination of chiral drugs arises from its greater potency and safety using fewer doses in comparison with racemates. ${ }^{4}$ Along with exponential development in the chiral drug market, regulatory policies have become more and more stringent. Thus, for the chiral drug to be approved, both enantiomers must be available and all eligibility studies have to be performed for both enantiomers separately. ${ }^{5}$

Asymmetric synthesis has vital access to enantiopure compounds; however, at the inception of chiral drug discovery, a plethora of molecules are required in milligram amount for pharmacological, pharmacokinetic, pharmacodynamic, and toxicological studies. Hence, based on cost and time factors, asymmetric synthesis is demonstrated as a substandard choice when compared to the resolution of racemates. ${ }^{6}$

Enantioselective liquid chromatography (LC) remains the most-preferred procedure either during the drug-discovery research process, to attain a few milligrams, or during the clinical trials, needing kilograms of material. ${ }^{7,8}$ LC demonstrated high stereorecognition ability, wide applicability, and high loading capacity, and a plethora of assorted commercial chiral stationary phases (CSPs) are offered. ${ }^{9,10}$ Among marketed
CSPs, polysaccharide-based CSPs were demonstrated as the most-efficient and widely used. Thus, more than $90 \%$ of the enantiomeric excess (ee) estimations were carried out employing chiral high-performance LC (HPLC) with polysaccharidebased CSPs. ${ }^{9,11,12}$ Carbamylated amylose and cellulose are the main backbone of polysaccharide-based CSPs. Despite the similarity of amylose- and cellulose-building units, their supramolecular arrangement is dissimilar. The cavity of amylose is helical, whereas cellulose is straight polymer chains. ${ }^{13,14}$ Also, there are differences between them in entropy and enthalpy changes in enantioselective adsorption. Cellulose-based columns showed enthalpy driven with negative entropic contribution but amylose-based columns exhibited positive entropic contribution. $^{15}$

It is documented that introducing analytical stereoselective separations of enantiomers using HPLC is very beneficial for monitoring asymmetric chemical reactions and enantioselective pharmacological and toxicological screening and is eligible for

Received: August 24, 2021

Accepted: September 15, 2021

Published: September 23, 2021 

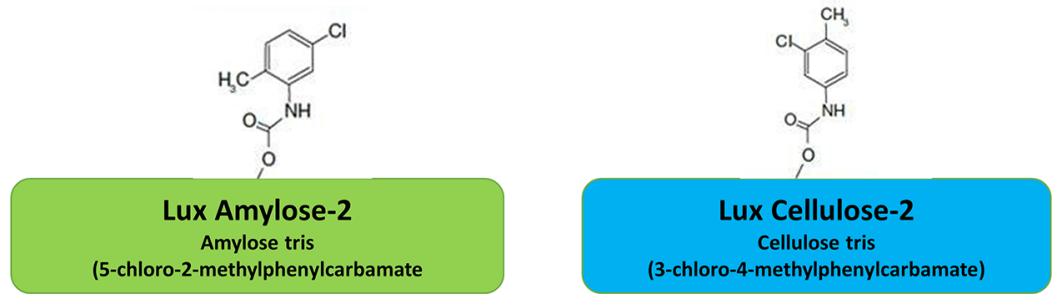

Figure 1. Chiral selectors of stationary phases Lux amylose-2 and Lux cellulose-2.

further optimization to achieve preparative HPLC techniques. $^{12,13,16}$

An integrated understanding of the molecular bases in chiral recognition will be achieved via further investigations on diverse sets of compounds and chiral selectors. Thus, recent investigations on HPLC enantioseparation of chiral compounds on polysaccharide-based CSPs under versatile mobile phase composition were conducted and resulted in a better understanding of chiral recognition. ${ }^{17-19}$ Compounds $\mathbf{1 - 1 8}$ have been synthesized in our group and showed promising biological activity. ${ }^{20}$ However, they have been tested in the racemic form; thus, it was of interest to estimate the biological activity of each enantiomer separately to study the impact of chirality on biological activity. Therefore, separation of the enantiomers of these compounds becomes a prerequisite to biological evaluation. Also, the outcomes of biological evaluation of each enantiomer separately will be followed with structure-activity relationship study to show the importance of the chiral center; otherwise, the pyrazoline moiety will be replaced with other moieties that have no chiral centers (e.g., pyrazole).

In the same vein, the potential of polysaccharide-based chiral columns (Lux amylose-2 and cellulose-2, Figure 1) and different mobile-phase modes (normal and polar organic modes) in selectivity and recognition of enantiomers was investigated via the development of stereoselective HPLC separations of the anticonvulsant 4,5-dihydro- $1 H$-pyrazole derivatives $(\mathbf{1}-\mathbf{1 8}){ }^{20}$ Moreover, the developed stereoselective analytical HPLC techniques of $\mathbf{1 - 1 8}$ will be applied in the stereospecific pharmacological, pharmacokinetic, pharmacodynamic, and toxicological studies of the bioactive compounds (1-18). Also, these stereoselective analytical HPLC separations could be utilized in monitoring stereoselective synthesis or to develop preparative HPLC techniques for these compounds.

Green analytical chemistry (GAC) defines a green analytical technique as one that eliminates environmental toxicity. ${ }^{21}$ Most pharmaceutical analyses are conducted in the pharmaceutical industry using highly flammable toxic solvents as the mobile phase. $^{22}$ The process is called a green analytical method if these toxic solvents are fully or partially substituted with green solvents, according to the GAC theory. ${ }^{23,24}$

\section{RESULTS AND DISCUSSION}

The mechanism of chiral separation is not yet fully recognized. However, many reports documented that solute-CSP interactions are the major factor that affects enantiomeric discrimination. Expected interactions between the solute and the chiral selector groups attached to the polysaccharide are hydrogen-bonding, dipole-dipole, and $\pi-\pi$ interactions. ${ }^{9,11,13}$ Based on the prementioned theory, the most important groups in our compounds that are expected to affect the chiral separation are $\mathrm{OCH}_{2} \mathrm{O}$ of benzendioxol, $\mathrm{N}-\mathrm{N}$ of pyrazoline, and carboxyhydrazide $(\mathrm{O}=\mathrm{CNHNHC}=\mathrm{O})$, hydrazone $(\mathrm{O}=$
$\mathrm{CNHN}=\mathrm{C}$ ), and oxadiazole at pyrazoline N-1. Also, the geometric features-size, shape, and position of the functional groups-of analytes will critically contribute to chiral recognition. In our discussion, we are focusing on the variable sidechain substituents at pyrazoline $\mathrm{N}-1$ (carboxyhydrazide $(\mathrm{O}=$ $\mathrm{CNHNHC}=\mathrm{O})$, hydrazone $(\mathrm{O}=\mathrm{CNHN}=\mathrm{C})$, and oxadiazole moieties) since it is the structural variation between compounds 1-18 and it should be responsible for differences in chromatographic elution and enantiomeric discrimination. Thus, our compounds were subdivided into three groups based on the side chain (Figure 2). The first group comprises compounds 1-8

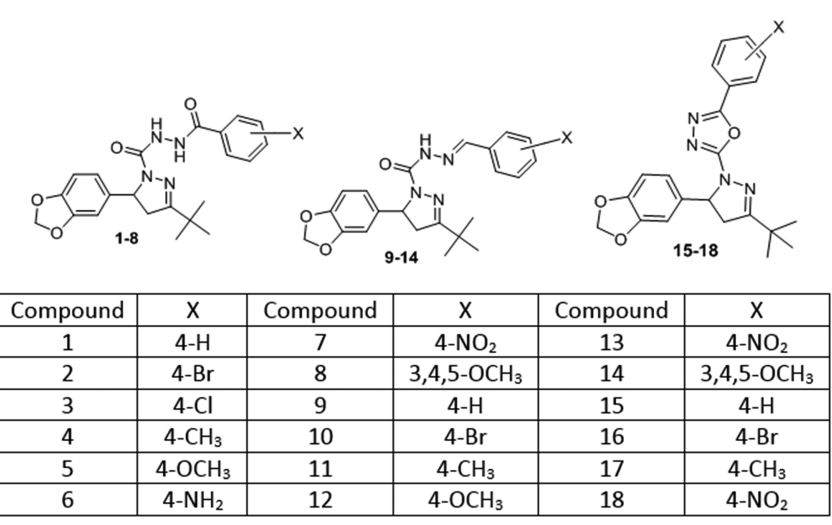

Figure 2. Chemical structures of compounds 1-18.

with a carboxyhydrazide $(\mathrm{O}=\mathrm{CNHNHC}=\mathrm{O})$ group which has two H-bond-donating $(2 \mathrm{NH})$ and two H-bond accepting $(2 \mathrm{C}=\mathrm{O})$ groups. The second group encloses compounds 9-14 with a hydrazone $(\mathrm{O}=\mathrm{CNHN}=\mathrm{C})$ moiety offering one $\mathrm{H}$ donating $(\mathrm{NH})$ and two $\mathrm{H}$-accepting $(\mathrm{C}=\mathrm{O}$ and $\mathrm{C}=\mathrm{N})$ groups, whereas the third group will be represented by compounds 15-18 having a 1,3,4-oxadiazole ring with three $\mathrm{H}$-accepting atoms $(\mathrm{O}$ and $2 \mathrm{C}==\mathrm{N})$.

Two elution modes were investigated in this work. First, the normal elution mode represented by $n$-hexane/ethanol has been principally emphasized as the most useful elution mode for polysaccharide-based CSPs. ${ }^{16}$ The second elution mode is the polar organic which has been well-established for analytical and preparative chiral separation. ${ }^{14}$ Many advantages have been documented of the polar organic mode counting short analysis time, high plate numbers, favorable peak shape, and high solubility of the solute in the mobile phase. ${ }^{16}$ Good solubility of the analyte in the mobile phase is crucial for our work since we are planning to utilize preparative separations of our compound's enantiomers in future work.

Normal Elution Using $n$-Hexane/Ethanol $(1: 1, v / v)$. The $n$-hexane/ethanol mixture has been selected based on previous reports that ethanol $(\mathrm{EtOH})$ as a polar modifier provides a higher success rate and better resolutions than isopropanol 


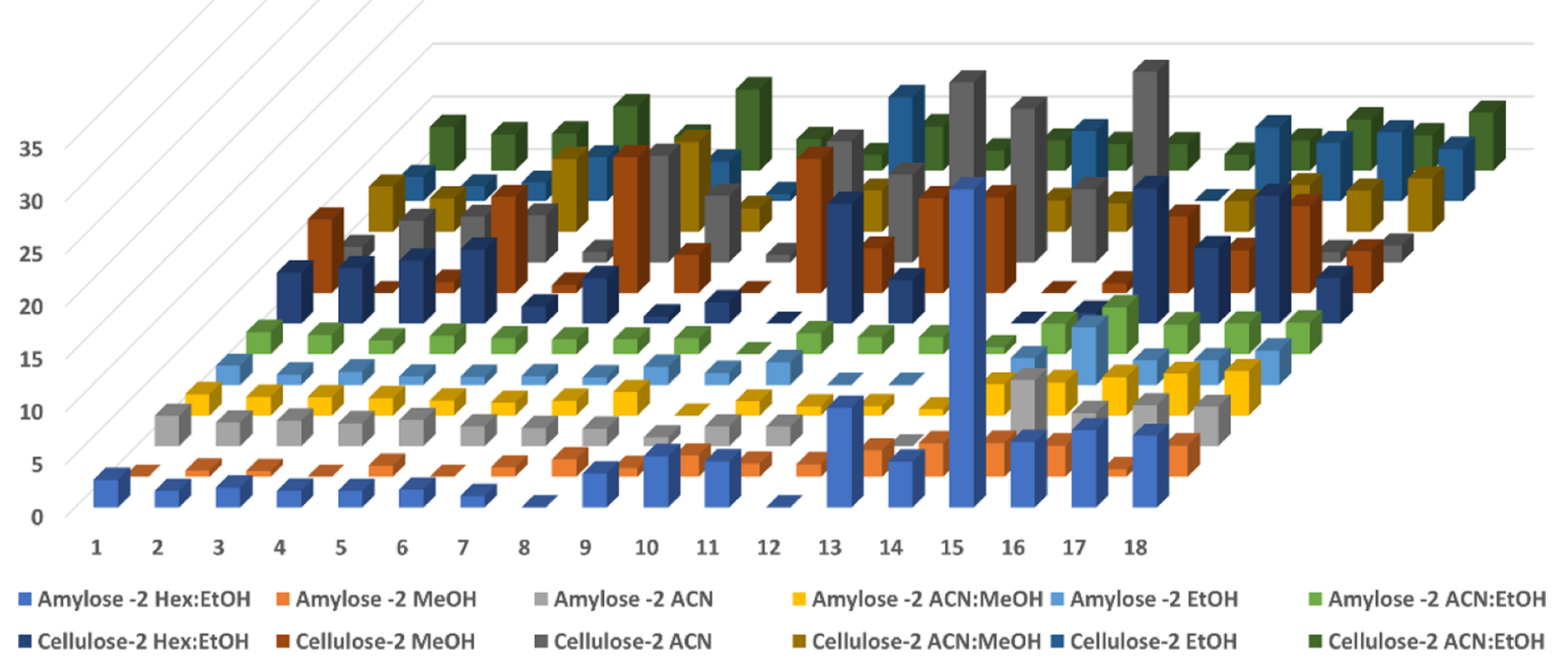

Figure 3. Resolution values $\left(R_{\mathrm{s}}\right)$ for tested compounds $(\mathbf{1 - 1 8})$ on Lux cellulose-2 and Lux amylose-2 CSPs, using different mobile phases.

(IPA) on such a set of stationary phases. ${ }^{13,25}$ In the normal elution mode, hydrogen-bonding between the analyte and the chiral selector is the most-significant contributor to chiral recognition. ${ }^{16}$ That is why an impressive effect has been noticed when altering the alcohol modifier ratio within the hydrocarbonbased mobile phases. Indeed, the protic alcohol modifier (having $\mathrm{OH}$ group) is competing with the solute for $\mathrm{H}$-bond-accepting groups in the chiral selector (phenyl carbamate-derivatized polysaccharide). Accordingly, the more the $\mathrm{H}$-donor groups in the solute, the more the enantiomeric discrimination of this solute will be negatively affected by the presence of an alcoholic modifier. This was reflected in the chiral recognition of compounds 1-18 in the normal elution mode, as seen in Figure 3. Group 3 (compounds 15-18, having only H-accepting groups in an oxadiazole moiety) showed overall better separation and higher resolution values - on both amyloseand cellulose-based CSP-than group 2 (compounds 9-14, having one $\mathrm{H}$-donor group and two $\mathrm{H}$-accepting groups) and at last was group 1 (compounds $1-8$, two $\mathrm{H}$-donor groups and two $\mathrm{H}$-accepting groups). These observations are typically matching the prementioned postulation. Except for compound 12, all compounds were baseline-separated on either cellulose- or amylose-based CSPs. Cellulose-based CSPs were superior to amylose-based CSPs in most cases (except for compound 15, Figure 3). Resolution values ranged from 1 to 30 and run time was $10-30 \mathrm{~min}$. As noticed, the more the $\mathrm{H}$-donor groups in the compound, the lesser the relative resolution. Further differences inside each group could be retrieved to geometric features and nonspecific interactions. ${ }^{11}$ Basic chromatographic parameters [resolution values $\left(R_{\mathrm{s}}\right)$, selectivity factor $(\alpha)$, and retention time $(t)$ ] for the tested compounds on both Lux cellulose-2 and Lux amylose- 2 column using hexane/ethanol in the ratio of $1: 1, \mathrm{v} / \mathrm{v}$ as the mobile phase are shown in Table S1 and Figure S1.

Polar Organic Elution Mode. As been mentioned in the above premises, a polar organic mode is very interesting for analytical and preparative chiral separation. ${ }^{17,18}$ In this work, we are interested in short runs and sharp peaks to be used later for monitoring the asymmetric synthesis of compounds $\mathbf{1 - 1 8}$ or to optimize preparative HPLC techniques to attain our compounds in the enantiopure form. Thus, five different mobile phases (ethanol, methanol, acetonitrile, or mixtures thereof) under the umbrella of the polar organic elution mode have been investigated.

Enantioseparation Using 100\% Methanol. Using methanol as the mobile phase, cellulose is still showing better resolution than amylose (Figure 3). Resolution values are 1-13 and run times are ranging from 5 to $10 \mathrm{~min}$. Despite the outlier in the normal elution mode (compound 15), no significant differences in resolution values between the normal mode and methanol have been observed. On the contrary to the normal elution mode, no special pattern has been followed by each group of our compounds using pure methanol as the mobile phase. Methanol's powerful solvating abilities and the $\mathrm{OH}$ group are expected to alter the capability of the solute to interact with the stationary phase and then accelerate the elution and lower the resolution of compounds 1-18, and the chiral discrimination is mostly due to geometric features and nonspecific interactions with the stationary phase. However, changing the polarity and the density of the mobile phase deviates from the three-dimensional structure of the stationary phase. ${ }^{11,13}$ Also, the decrease in the mobile-phase viscosity rushes the solute diffusion in the mobile phase, reducing band broadening which causes an increase in column efficiency. ${ }^{12}$ This will neutralize the negative impact of methanol's powerful solvating abilities and the $\mathrm{OH}$ group on the chiral recognition of compounds 1-18. Thus, all compounds were baseline-separated with very sharp peaks in short time runs, which is perfect for reaction monitoring and optimization of preparative techniques. Short time runs are an expected consequence of the increase in mobile-phase polarity and decrease in viscosity. Basic chromatographic parameters [resolution $\left(R_{\mathrm{s}}\right)$, selectivity factor $(\alpha)$, and retention time $(t)]$ for the tested compounds on both Lux cellulose- 2 and Lux amylose- 2 columns using $100 \%$ methanol as the mobile phase are shown in Table S2 and Figure S2. 
Table 1. Resolution Values $\left(R_{s}\right)$ for Tested Compounds 1-18 on the Lux Cellulose-2 Column and Lux Amylose-2 CSPs, Using Different Mobile Phases

\begin{tabular}{|c|c|c|c|c|c|c|c|c|c|c|c|c|}
\hline \multirow{3}{*}{$\begin{array}{c}\text { column } \\
\text { compound }\end{array}$} & \multicolumn{12}{|c|}{ mobile phase } \\
\hline & \multicolumn{6}{|c|}{ amylose-2 } & \multicolumn{6}{|c|}{ cellulose-2 } \\
\hline & $\begin{array}{c}\mathrm{MeOH} \\
100 \%\end{array}$ & $\begin{array}{c}\mathrm{ACN} / \mathrm{MeOH} \\
1 / 1 \mathrm{v} / \mathrm{v}\end{array}$ & $\begin{array}{l}\mathrm{ACN} \\
100 \%\end{array}$ & $\begin{array}{c}\mathrm{ACN} / \mathrm{EtOH} \\
1 / 1 \mathrm{v} / \mathrm{v}\end{array}$ & $\begin{array}{l}\text { EtOH } \\
100 \%\end{array}$ & $\begin{array}{c}n-\mathrm{Hex} / \mathrm{EtOH} \\
1 / 1 \mathrm{v} / \mathrm{v}\end{array}$ & $\begin{array}{c}\mathrm{MeOH} \\
100 \%\end{array}$ & $\begin{array}{c}\mathrm{ACN} / \mathrm{MeOH} \\
1 / 1 \mathrm{v} / \mathrm{v}\end{array}$ & $\begin{array}{l}\mathrm{ACN} \\
100 \%\end{array}$ & $\begin{array}{c}\mathrm{ACN} / \mathrm{EtOH} \\
1 / 1 \mathrm{v} / \mathrm{v}\end{array}$ & $\begin{array}{l}\text { EtOH } \\
100 \%\end{array}$ & $\begin{array}{c}n-\mathrm{Hex} / \mathrm{EtOH} \\
1 / 1 \mathrm{v} / \mathrm{v}\end{array}$ \\
\hline 1 & NS & 2.03 & 2.90 & 2.11 & 1.82 & 2.61 & 6.97 & 4.27 & 1.47 & 4.14 & 2.29 & 4.84 \\
\hline 2 & 0.59 & 1.78 & 2.24 & 1.84 & 0.99 & 1.58 & NS & 3.17 & 3.97 & 3.44 & 1.38 & 5.28 \\
\hline 3 & 0.56 & 1.76 & 2.43 & 1.32 & 1.26 & 1.89 & 1.05 & 3.28 & 4.33 & 3.53 & 1.77 & 5.97 \\
\hline 4 & NS & 1.65 & 2.18 & 1.74 & 0.80 & 1.59 & 9.11 & 6.89 & 4.45 & 6.09 & 4.14 & 7.04 \\
\hline 5 & 1.07 & 1.42 & 2.48 & 1.51 & 0.77 & 1.58 & 0.78 & 2.98 & 1.00 & 3.21 & 0.79 & 1.65 \\
\hline 6 & NS & 1.22 & 1.91 & 1.42 & 0.85 & 1.71 & 12.90 & 8.52 & 10.12 & 7.70 & 3.73 & 4.32 \\
\hline 7 & 0.91 & 1.40 & 1.71 & 1.40 & 0.73 & 1.09 & 3.60 & 2.24 & 6.31 & 2.97 & 0.68 & 0.68 \\
\hline 8 & 1.68 & 2.24 & 1.67 & 1.50 & 1.73 & NS & NS & 1.65 & 0.72 & 1.46 & NS & 2.05 \\
\hline 9 & 0.84 & NS & 0.84 & NS & 1.13 & 3.23 & 12.72 & 3.92 & 11.49 & 4.18 & 9.86 & NS \\
\hline 10 & 2.03 & 1.35 & 1.90 & 1.99 & 2.15 & 4.88 & 4.22 & 1.76 & 8.33 & 1.90 & 3.44 & 11.37 \\
\hline 11 & 1.24 & 0.88 & 1.86 & 1.62 & NS & 4.38 & 9.01 & 3.06 & 17.08 & 2.84 & 6.92 & 4.12 \\
\hline 12 & 1.2 & 0.89 & 1.94 & 1.62 & NS & NS & 9.08 & 2.98 & 14.58 & 2.53 & 6.66 & NS \\
\hline 13 & 3.19 & 2.97 & 2.36 & 2.90 & 2.53 & 4.35 & 0.92 & 1.06 & 18.10 & 1.52 & NS & 1.04 \\
\hline 14 & 2.50 & 0.60 & NS & 0.69 & 2.17 & 9.48 & NS & 2.68 & 6.93 & 2.51 & NS & NS \\
\hline 15 & 3.22 & 3.15 & 6.27 & 4.47 & 5.47 & 30.21 & 7.26 & 2.91 & 0.62 & 2.84 & 6.98 & 12.85 \\
\hline 16 & 2.97 & 3.66 & 3.16 & 2.84 & 2.32 & 6.23 & 4.07 & 4.41 & 0.60 & 4.83 & 5.52 & 7.17 \\
\hline 17 & 0.72 & 3.98 & 3.90 & 2.94 & 2.36 & 7.34 & 8.26 & 3.89 & 0.99 & 3.32 & 6.55 & 12.12 \\
\hline 18 & 2.97 & 4.20 & 3.74 & 2.99 & 3.26 & 6.82 & 3.97 & 5.06 & 1.60 & 5.49 & 4.95 & 4.32 \\
\hline
\end{tabular}

Enantioseparation Using $100 \%$ Acetonitrile. Acetonitrile is a powerful solvent like methanol; however, there is a major difference between them. Acetonitrile is an aprotic solvent with the $\mathrm{CN}$ group which is a $\mathrm{H}$-bond-accepting group not like the $\mathrm{H}$-donating $\mathrm{OH}$ group of the protic solvent methanol. These differences are expected to have influences on chiral recognition abilities of the stationary phase using acetonitrile as the mobile phase. As expected from $\mathrm{H}$-bond competition postulation, group 3 is the most-affected group after the use of acetonitrile as the mobile phase (Figure 3). The resolution of group 3 (compounds 15-18) has been greatly moved to lower values in comparison with both $n$-hexane/ethanol and methanol. Dissimilar to the protic polar solvent (methanol and ethanol), acetonitrile $\left(\mathrm{CH}_{3} \mathrm{CN}\right)$ is expected to compete with group 3 members for $\mathrm{H}$-donating groups in the stationary phase; thus, it will interfere with the formation of a solute-CSP complex which is responsible for chiral separation (Figure S5). On the other hand, group 2 (compounds 9-14) showed very good resolutions using pure acetonitrile as a mobile phase and was better than group 1 (compounds 1-8). All compounds were baseline-separated either on amylose- or cellulose-based CSPs or both. Cellulose is still superior to amylose for either group 1 or 2, but for group 3, amylose displayed higher resolution values using pure acetonitrile. Resolution ranged from 1 to 18 and runs times were 5-30 min. Basic chromatographic parameters [resolution $\left(R_{\mathrm{s}}\right)$, selectivity factor $(\alpha)$, and retention time $(t)$ ] for the tested compounds on both Lux cellulose- 2 and Lux amylose- 2 columns using $100 \%$ acetonitrile as the mobile phase are shown in Table S3 and Figure S3.

Enantioseparation Using Methanol/Acetonitrile (1:1 $\mathbf{v} / \mathbf{v})$. Using a binary mixture of methanol/acetonitrile did not affect the resolution of group 1 (Figure 3). However, a great change in groups 2 and 3 occurred especially on cellulose-based CSPs. Resolution values for group 2 lowered from 10 to 18 using acetonitrile $100 \%$ to $1-4$ using methanol/acetonitrile $1 / 1 \mathrm{v} / \mathrm{v}$. While for group 3, resolution improved for all compounds on the cellulose column. The overall impact of altering mobile-phase composition is not positive. The presence of both protic and aprotic solvents in the mobile phase weakened all possible $\mathrm{H}$ bond interactions between the solute and the stationary phase resulting in inappropriate chiral recognition. Thus, the resolution values range is $1-8$ which is inferior to that observed using either pure acetonitrile or pure methanol. The only benefit was the improvement in resolution of group 3 on cellulose and the impressive change in run time $(3-6 \mathrm{~min})$ to be tremendously short with very sharp and symmetric peaks. Thus, this mobile phase should be considered for reaction monitoring. All compounds were baseline-separated using this mobile phase and the cellulose column still showing overall dominance compared to amylose. Basic chromatographic parameters [resolution $\left(R_{\mathrm{s}}\right)$, selectivity factor $(\alpha)$, and retention time $(t)]$ for the tested compounds on both Lux cellulose- 2 and Lux amylose- 2 columns using methanol/acetonitrile in the ratio of $1: 1 \mathrm{v} / \mathrm{v}$ as the mobile phase are shown in Table $S 4$ and Figure S4.

Enantioseparation Using $100 \%$ Ethanol. As has been noticed with methanol, the pure ethanol mobile phase was worthy for group 3 having only $\mathrm{H}$-accepting groups and group 2 with only one $\mathrm{H}$-donor group. Group 1 showed the lowest resolution which is explained by the competition of ethanolic $\mathrm{OH}$ with group 1 solute's NHs on the stationary phase. Cellulose is enduring its higher resolution compared to amylose. The resolution values were $1-10$ and run time ranged from 8 to 35 min for amylose and 7-17 min for cellulose. Slightly delayed runs of ethanol compared to methanol is a consequence to the change in mobile-phase polarity and viscosity which moreover imitated as a slight variation in resolution values due to the decrease in column efficacy following slight viscosity increase, as shown in Figure 3. Basic chromatographic parameters [resolution $\left(R_{\mathrm{s}}\right)$, selectivity factor $(\alpha)$, and retention time $(t)$ ] for the tested compounds on both Lux cellulose-2 and Lux amylose- 2 columns using $100 \%$ ethanol as the mobile phase are shown in Table S5 and Figure S5.

Enantioseparation Using Ethanol/Acetonitrile (1:1 v/ v). Similarly to the methanol/acetonitrile mixture, the ethanol/ acetonitrile mobile phase showed almost typical results to that of 
A

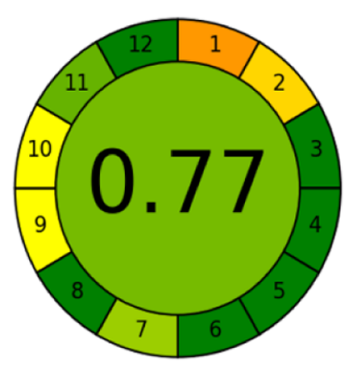

D

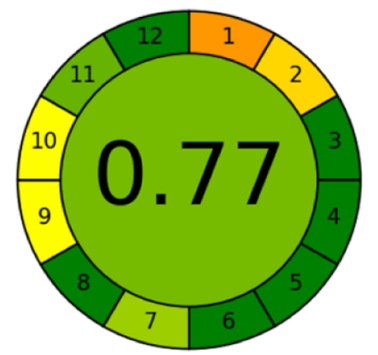

B

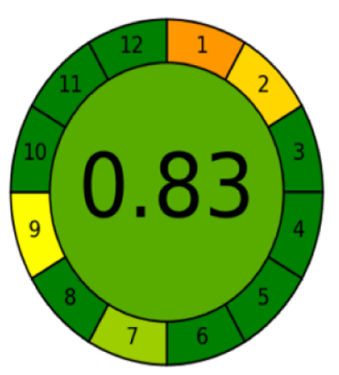

$\mathbf{E}$

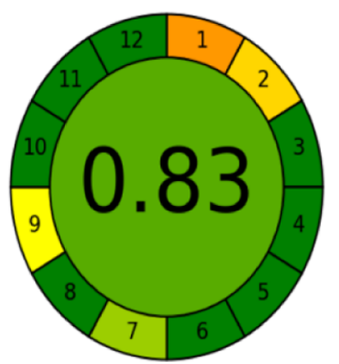

C

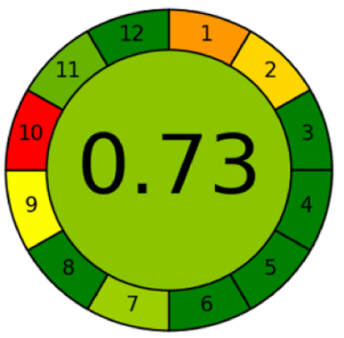

F

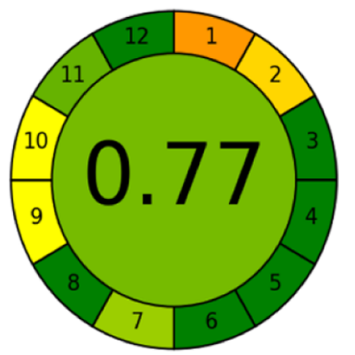

Figure 4. AGREE analytical score after using different mobile phases: (A) hexane/ethanol, (B) methanol, (c) acetonitrile, (d) methanol/acetonitrile, (e) ethanol, and (f) ethanol/acetonitrile.

methanol/acetonitrile (Figure 3). Resolution values and run time for both mobile phases were almost identical. Interestingly, compound 9 has been resolved only on cellulose using both mobile phases with almost equal resolution $\left(R_{\mathrm{s}} 3.92\right.$ using methanol/acetonitrile and 4.12 using ethanol/acetonitrile). The consistency of methanol/acetonitrile and ethanol/acetonitrile results confirms the integrity of the postulated theory. Basic chromatographic parameters [resolution $\left(R_{\mathrm{s}}\right)$, selectivity factor $(\alpha)$, and retention time $(t)]$ for the tested compounds on both Lux cellulose- 2 and Lux amylose- 2 columns using ethanol/ acetonitrile in the ratio of $1: 1(\mathrm{v} / \mathrm{v})$ as the mobile phase are shown in Table S6 and Figure S6.

General Comparative Enantioseparation. As per Table 1 and Figure 3, the Lux cellulose-2 column has proved supreme chiral recognition ability to all compounds translated into higher-resolution values compared to the Lux amylose-2 column. With the Lux cellulose- 2 column, $n$-hexane/ethanol is the best mobile phase for group 3 (compounds 15-18) and acetonitrile is the best one for group 2 (compounds 9-14). Concerning group 1 , methanol was the best mobile phase for compounds 1, 4, 6, and 9, $n$-hexane/ethanol was the best mobile phase for compounds $\mathbf{2 , 3}$, and $\mathbf{8}$, acetonitrile for compound 7 , and acetonitrile/ethanol or methanol binary mixtures for compound $\mathbf{5}$.

The amylose- 2 column was more suitable for the normal elution mode. The hexane/ethanol mobile phase was superior for all members of group 2 and 3 (compounds 9-18) and was a potent rival for other mobile phases for group 1 (compounds 18) using an amylose- 2 column. The fact that the Lux amylose- 2 column exhibits better column efficacy under low-polarity mobile phases is well-documented. ${ }^{12,26}$ On the contrary, the polar organic elution mode looks to be more suitable for a cellulose-2 column. Highly polar mobile-phase compositions have proved marvelous recognition ability on the Lux cellulose-2 column (Figure S10). This phenomenon has been documented in the present work and also has been reported in the former work. $^{12,26}$ The dissimilar supramolecular arrangements of the polysaccharides (amylose is helical, whereas cellulose is straight polymer chains ${ }^{14}$ ) should have a contribution to the diversity in behavior of amylose- and cellulose-based CSPs under versatile mobile-phase polarity.

HPLC chromatograms of compounds $\mathbf{1 - 1 8}$ on Lux amylose2 and Lux cellulose- 2 using different mobile phases are shown in Figures S7 and S8.

Greenness Profile Evaluation. In this study, the greenness of different elution modes for enantiomeric separation of 18 biologically active racemic 4,5-dihydro- $1 H$-pyrazole derivatives was obtained using the "AGREE Analytical Greenness Calculator (version 0.5, Gdansk University of Technology, Gdansk, Poland, 2020)". This metric system considered all 12 principles of GAC and offered several advantages over other methods of greenness evaluation. ${ }^{24}$ The AGREE analytical scores of different mobile phases used were $0.77,0.83,0.73,0.77$, 0.83 , and 0.77 for hexane/ethanol ( $1: 1 \mathrm{v} / \mathrm{v})$, methanol $(100 \%)$, acetonitrile $(100 \%)$, methanol/acetonitrile $(1: 1 \mathrm{v} / \mathrm{v})$, ethanol (100\%), and ethanol/acetonitrile $(1: 1, \mathrm{v} / \mathrm{v})$; respectively, as shown in Figure 4.

The AGREE analytical scores above 0.75 indicated excellent green analytical methodology for the drug analysis. However, the AGREE analytical score of 0.50 indicated that the method is acceptable for drug analysis. On the other hand, the AGREE analytical scores below 0.50 showed the unacceptability of the analytical technique. $^{24}$

Based on AGREE analytical scores obtained for different elution systems, both methanol (100\%) and ethanol (100\%) were considered excellent green solvents for the enantiomeric separation, especially when compared with the normal elution mode using hexane as an eluting solvent. Analytical greenness sheet reports for the different elution solvents are shown in Figures S9-S14. 


\section{CONCLUSIONS}

Based on a study of the enantioseparation of 18 pyrazoline derivatives on cellulose- and amylose-based chiral HPLC columns with one normal and five polar organic elution modes, the enantiomer-resolving ability of amylose-based columns was greater using the normal elution mode, whereas the cellulose-based column was superior with all polar organic mobile phases. Several baseline stereoselective HPLC methods have been developed for the tested compounds 1-18. This plethora of enantioselective methods will be utilized to monitor the stereoselective synthesis of compounds $\mathbf{1 - 1 8}$ or to develop preparative HPLC techniques for compounds 1-18, followed by stereospecific pharmacological studies for each enantiomer separately. The nature of mobile-phase components has greatly affected the chiral recognition of the stationary phase via competing for the solute for the stationary phase-binding groups. Greenness analytical scores were assessed where methanol and ethanol gave excellent green scores as mobilephase green solvents for enantiomeric resolving of the studied compounds.

\section{EXPERIMENTAL SECTION}

Chemicals and Reagents. Compounds from 1-18 have been synthesized according to the reported procedures by ElBehairy et al. ${ }^{20}$ HPLC-grade solvents ( $n$-hexane, methanol, ethanol, and acetonitrile) were purchased from Sigma-Aldrich (St. Louis, MO, USA). HPLC enantioseparation was performed using the commercially available Lux amylose-2 [amylose tris(5chloro-2-methylphenylcarbamate)] and Lux cellulose-2 [cellulose tris(3-chloro-4-methylphenylcarbamate)] $(250 \mathrm{~mm} \times 4.6$ $\mathrm{mm}, 3 \mu \mathrm{m}$ ) columns (Phenomenex, Le Pecq, France).

Instruments and Chromatographic Conditions. The HPLC apparatus used for analytical enantioseparations consisted of an HP automatic injector and a PDA detector (Agilent Technologies, Palo Alto, CA), an HP 1100 quaternary pump (Agilent Technologies), and a vacuum degasser. The signal was acquired and processed using HP ChemStation software. The analysis was performed at a temperature of $25^{\circ} \mathrm{C}$ with a mobile phase flow rate of $1 \mathrm{~mL} / \mathrm{min}$. The detection wavelength was set at $254 \mathrm{~nm}$.

Greenness Profile Evaluation. Using 12 different GAC principles, the greenness of the different elution modes used for the enantiomeric separation of the 18 biologically active racemic 4,5-dihydro- $1 \mathrm{H}$-pyrazole derivatives was calculated. PenaPereira et al. ${ }^{24}$ presented these GAC concepts. The greenness score for both the normal and polar organic elution modes was calculated using AGREE's analytical rating for "AGREE Analytical Greenness Calculator” (version 0.5, Gdansk University of Technology, Gdansk, Poland, 2020).

\section{ASSOCIATED CONTENT}

\section{SI Supporting Information}

The Supporting Information is available free of charge at https://pubs.acs.org/doi/10.1021/acsomega.1c04613.

Resolution $\left(R_{\mathrm{s}}\right)$, selectivity $(\alpha)$, and retention time $(t)$ values for tested compounds $\mathbf{1 - 1 8}$ on the Lux cellulose-2 column and Lux amylose-2 CSPs, using hexane/ethanol $(1: 1, \mathrm{v} / \mathrm{v})$ as the mobile phase; resolution $\left(R_{\mathrm{s}}\right)$, selectivity $(\alpha)$, and retention time $(t)$ values for tested compounds 1-18 on the Lux cellulose- 2 column and Lux amylose-2 CSPs, using $100 \%$ methanol as the mobile phase; resolution $\left(R_{\mathrm{s}}\right)$, selectivity $(\alpha)$, and retention time $(t)$ values for tested compounds $\mathbf{1 - 1 8}$ on the Lux cellulose-2 column and Lux amylose-2 CSPs, using 100\% acetonitrile as the mobile phase; resolution $\left(R_{\mathrm{s}}\right)$, selectivity $(\alpha)$, and retention time $(t)$ values for tested compounds $\mathbf{1 - 1 8}$ on the Lux cellulose- 2 column and Lux amylose-2 CSPs, using acetonitrile/methanol $(1: 1 \mathrm{v} / \mathrm{v})$ as the mobile phase; resolution $\left(R_{\mathrm{s}}\right)$, selectivity $(\alpha)$, and retention time ( $t$ ) values for tested compounds 1-18 on the Lux cellulose- 2 column and Lux amylose-2 CSPs, using 100\% ethanol as the mobile phase; resolution $\left(R_{\mathrm{s}}\right)$, selectivity $(\alpha)$, and retention time $(t)$ values for tested compounds 1-18 on the Lux cellulose-2 column and Lux amylose-2 CSPs, using acetonitrile/ethanol $(1: 1, \mathrm{v} / \mathrm{v})$ as the mobile phase; resolution values $\left(R_{\mathrm{s}}\right)$ for tested compounds $\mathbf{1 - 1 8}$ on Lux cellulose- 2 and Lux amylose-2 CSPs, using $n$ hexane/ethanol $(1 / 1 \mathrm{v} / \mathrm{v})$; resolution values $\left(R_{\mathrm{s}}\right)$ for tested compounds $1-18$ on Lux cellulose- 2 and Lux amylose-2 CSPs, using 100\% methanol; resolution values $\left(R_{\mathrm{s}}\right)$ for tested compounds $\mathbf{1 - 1 8}$ on Lux cellulose- 2 and Lux amylose-2 CSPs, using 100\% acetonitrile; resolution values $\left(R_{\mathrm{s}}\right)$ for tested compounds $\mathbf{1 - 1 8}$ on Lux cellulose2 and Lux amylose-2 CSPs, using acetonitrile/methanol $(1 / 1 \mathrm{v} / \mathrm{v})$; resolution values $\left(R_{\mathrm{s}}\right)$ for tested compounds 1-18 on Lux cellulose-2 and Lux amylose-2 CSPs, using $100 \%$ ethanol; resolution values $\left(R_{\mathrm{s}}\right)$ for tested compounds 1-18 on Lux cellulose- 2 and Lux amylose-2 CSPs, using acetonitrile/ethanol $1 / 1 \mathrm{v} / \mathrm{v}$; chromatograms of compounds 1-18 on Lux amylose-2 using different mobile phases; chromatograms of compounds $\mathbf{1 - 1 8}$ on Lux cellulose- 2 using different mobile phases; analytical greenness report sheet using hexane/ethanol (1:1) as the mobile phase; analytical greenness report sheet using $100 \%$ methanol as the mobile phase; analytical greenness report sheet using $100 \%$ acetonitrile as the mobile phase; analytical greenness report sheet using acetonitrile/ methanol (1:1) as the mobile phase; analytical greenness report sheet using $100 \%$ ethanol as the mobile phase; and analytical greenness report sheet using acetonitrile/ ethanol (1:1) as the mobile phase (PDF)

\section{AUTHOR INFORMATION}

\section{Corresponding Author}

Inas A. Abdallah - Department of Analytical Chemistry, Faculty of Pharmacy, University of Sadat City, Sadat City 32897, Egypt; 이이.org/0000-0002-4207-0985; Email: inas.abdallah@fop.usc.edu.eg

\section{Authors}

Mohammed Farrag El-Behairy - Department of Organic and Medicinal Chemistry, Faculty of Pharmacy, University of Sadat City, Sadat City 32897, Egypt; 이이이.org/0000001-7843-1423

Rasha M. Hassan - Medicinal and Pharmaceutical Chemistry Department, Pharmaceutical and Drug Industries Research Division, National Research Centre, Giza 12511, Egypt

Complete contact information is available at:

https://pubs.acs.org/10.1021/acsomega.1c04613

\section{Author Contributions}

Conceptualization, M.F.E.-B.; methodology, M.F.E.-B., R.M.H., and I.A.A.; investigation, M.F.E.-B. and I.A.A.; data curation, M.F.E.-B. and R.M.H; writing-original draft preparation, 
M.F.E.-B., R.M.H., and I.A.A.; writing-review and editing; M.F.E.-B., R.M.H., and I.A.A.; supervision, M.F.E.-B. and I.A.A.; project administration, R.M.H; and funding acquisition, M.F.E.B. and I.A.A. All authors have read and agreed to the published version of the manuscript.

\section{Notes}

The authors declare no competing financial interest.

\section{ACKNOWLEDGMENTS}

The authors acknowledge the Faculty of Pharmacy, University of Sadat City, Egypt, and the National Research Center, Egypt, for providing the required facilities to conduct this study.

\section{REFERENCES}

(1) Alkadi, H.; Jbeily, R. Role of Chirality in Drugs: An Overview. Infect. Disord.: Drug Targets 2018, 18, 88-95.

(2) Calcaterra, A.; D'Acquarica, I. The market of chiral drugs: Chiral switches versus de novo enantiomerically pure compounds. J. Pharm. Biomed. Anal. 2018, 147, 323-340.

(3) Urquhart, L. Top drugs and companies by sales in 2018. Nat. Rev. Drug Discovery 2019, 18, 245.

(4) Brocks, D. R. Drug disposition in three dimensions: an update on stereoselectivity in pharmacokinetics. Biopharm. Drug Dispos. 2006, 27, 387-406.

(5) FDA FDA's policy statement for the development of new stereoisomeric drugs. Chirality, 1992, 4, 338-340. DOI: 10.1002/ chir.530040513

(6) Toda, F. Enantiomer Separation, Fundamentals and Practical Methods; Springer Netherlands, 2004.

(7) Gumustas, M.; Ozkan, S. A.; Chankvetadze, B. Analytical and Preparative Scale Separation of Enantiomers of Chiral Drugs by Chromatography and Related Methods. Curr. Med. Chem. 2018, 25, 4152-4188.

(8) Pinto, M. M. M.; Fernandes, C.; Tiritan, M. E. Chiral Separations in Preparative Scale: A Medicinal Chemistry Point of View. Molecules 2020, 25, 1931-1947.

(9) Ikai, T.; Okamoto, Y. Preparation and Chiral Recognition of Polysaccharide-Based Selectors. Chiral Recognition in Separation Methods; Springer, 2010.

(10) Chankvetadze, B. Recent trends in preparation, investigation and application of polysaccharide-based chiral stationary phases for separation of enantiomers in high-performance liquid chromatography. Trends Anal. Chem. 2020, 122, 115709-115722.

(11) Okamoto, Y.; Yashima, E. Polysaccharide Derivatives for Chromatographic Separation of Enantiomers. Angew. Chem., Int. Ed. 1998, 37, 1020-1043.

(12) Toribio, L.; del Nozal, M. J.; Bernal, J. L.; Bernal, J.; Martín, M. T. Study of the enantiomeric separation of an acetamide intermediate by using supercritical fluid chromatography and several polysaccharide based chiral stationary phases. J. Chromatogr. A 2011, 1218, 48864891.

(13) El-Behairy, M. F.; El-Azzouny, A. A. Enantioselective HPLC separation of bioactive C5-chiral 2-pyrazolines on lux amylose-2 and lux cellulose-2: Comparative and mechanistic approaches. J. Liq. Chromatogr. Relat. Technol. 2016, 39, 346-353.

(14) Winger, M.; Christen, M.; van Gunsteren, W. F. On the conformational properties of amylose and cellulose oligomers in solution. Int. J. Carbohydr. Chem. 2009, 2009, 1-8.

(15) Kazusaki, M.; Shoda, T.; Kawabata, H. Enthalpy-entropy compensation for enantio-separation on cellulose and amylose tris (3, 5-dimethylphenylcarbamate) derivatives as stationary phases. Chromatography 2003, 24, 121-126.

(16) Gogaladze, K.; Chankvetadze, L.; Tsintsadze, M.; Farkas, T.; Chankvetadze, B. Effect of Basic and Acidic Additives on the Separation of Some Basic Drug Enantiomers on Polysaccharide-Based Chiral Columns With Acetonitrile as Mobile Phase. Chirality 2015, 27, 228234.
(17) Panusa, A.; Rosetti, A.; Villani, C.; Cirilli, R. Direct HPLC enantioseparation of chemopreventive chiral isothiocyanates sulforaphane and iberin on immobilized amylose-based chiral stationary phases under normal-phase, polar organic and aqueous conditions. Talanta 2020, 218, 121151-121157.

(18) Peluso, P.; Sechi, B.; Lai, G.; Dessì, A.; Dallocchio, R.; Cossu, S.; Aubert, E.; Weiss, R.; Pale, P.; Mamane, V.; Chankvetadze, B. Comparative enantioseparation of chiral 4,4'-bipyridine derivatives on coated and immobilized amylose-based chiral stationary phases. $J$. Chromatogr. A 2020, 1625, 461303-461312.

(19) Peluso, P.; Chankvetadze, B. The molecular bases of chiral recognition in 2-(benzylsulfinyl)benzamide enantioseparation. Anal. Chim. Acta 2021, 1141, 194-205.

(20) El-behairy, M. F.; Attia, H. N. H. Design, synthesis and anticonvulsant profile of 5-(benzo [D] [1,3] dioxol-5-yl)-3-tert-butyl-4, 5-dihydropyrazole. Int. J. Pharm. Pharm. Sci. 2017, 9, 180-188.

(21) Sheldon, R. A. Green solvents for sustainable organic synthesis: state of the art. Green Chem. 2005, 7, 267-278.

(22) Garrigues, S.; Armenta, S.; Guardia, M. D. L. Green strategies for decontamination of analytical wastes. Trends Anal. Chem. 2010, 29, 592-601.

(23) Anastas, P. T.; Warner, J. C. Green Chemistry: Theory and Practice; Oxford University Press: New York, 1998.

(24) Pena-Pereira, F.; Wojnowski, W.; Tobiszewski, M. AGREEAnalytical GREEnness Metric Approach and Software. Anal. Chem. 2020, 92, 10076-10082.

(25) Younes, A. A.; Mangelings, D.; Vander Heyden, Y. Chiral separations in normal phase liquid chromatography: enantioselectivity of recently commercialized polysaccharide-based selectors. Part I: enantioselectivity under generic screening conditions. J. Pharm. Biomed. Anal. 2011, 55, 414-423.

(26) West, C.; Bouet, A.; Gillaizeau, I.; Coudert, G.; Lafosse, M.; Lesellier, E. Chiral separation of phospine-containing $\alpha$-amino acid derivatives using two complementary cellulosic stationary phases in supercritical fluid chromatography. Chirality 2010, 22, 242-251. 